\title{
Expressions and significances of the angiotensin-converting enzyme 2 gene, the receptor of SARS-CoV-2 for COVID-19
}

\author{
Jiewen $\mathrm{Fu}^{1} \cdot$ Baixu Zhou ${ }^{1,2} \cdot$ Lianmei Zhang $^{1,3} \cdot$ Kyathegowdanadoddi Srinivasa Balaji $^{4}{ }^{\circ} \cdot$ Chunli Wei $^{1} \cdot$ Xiaoyan Liu $^{1}$. \\ Hanchun $\mathrm{Chen}^{5}$. Jiangzhou Peng ${ }^{6}$. Junjiang Fu ${ }^{1}$ (1)
}

Received: 25 March 2020 / Accepted: 28 April 2020 / Published online: 14 May 2020

(c) Springer Nature B.V. 2020

\begin{abstract}
The ACE2 gene is a receptor of SARS-CoV-2 (severe acute respiratory syndrome coronavirus 2) for COVID-19 (coronavirus disease 2019). To analyze the expression profiles and clinical significances for this gene in humans, RNA-seq data representing 27 different tissues were analyzed using NCBI; total RNA was extracted from different tissues of mouse and semi-quantitative reverse transcriptional-polymerase chain reaction (Q-RT-PCR) was carried out. Immunohistochemistry expression profiles in normal tissues and cancer tissues and TCGA survival analysis in renal and liver cancer were conducted. ACE2 was highly conserved in different species. In normal tissues, ACE2 expression distributions were organ-specific, mainly in the kidney, male testis and female breast, and cardiovascular and gastrointestinal systems. High level of expression in testis, cardiovascular and gastrointestinal system indicated that SARS-CoV-2 might not only attack the lungs, but also affect other organs, particularly the testes, thus it may severely damage male sexual development for younger male and lead to infertility in an adult male, if he contracted COVID-19. On the other side, high expression of ACE2 was correlated with increased survival rate in renal and liver cancer, indicating that $A C E 2$ is a prognostic marker in both renal cancer and liver cancers. Thus, the ACE2 is a functional receptor for SARS-CoV-2 and has a potential anti-tumor role in cancer. Taken together, this study may not only provide potential clues for further medical pathogenesis of COVID-19 and male fertility, but also indicate the clinical significance of the role of the ACE2 gene in cancer.
\end{abstract}

Keywords ACE2 gene $\cdot$ SARS-CoV-2 $\cdot$ COVID-19 $\cdot$ RNA-sequencing $\cdot$ Immunohistochemistry (IHC) $\cdot$ Testis $\cdot$ Cancer

Jiewen Fu, Baixu Zhou and Lianmei Zhang have equally contributed.

Jiangzhou Peng

jiangzhou83@163.com

$\triangle$ Junjiang Fu

fujunjiang@hotmail.com

1 Key Laboratory of Epigenetics and Oncology, the Research Center for Preclinical Medicine, Southwest Medical University, Luzhou 646000, Sichuan Province, China

2 Department of Gynecology and Obstetrics, Guangdong Women and Children Hospital, Guangzhou, Guangdong, China

3 Department of Pathology, The Affiliated Huaian No. 1 People's Hospital of Nanjing Medical University, Huai'an, China

\section{Introduction}

The Angiotensin-converting enzyme 2 gene (ACE2) (OMIM: 300335), also known as ACEH, is attached to the cell membrane of the outer layer tissues of the heart, arteries,

4 Post Graduation Department of Studies and Research in Biotechnology, Teresian Research Center, Teresian College (Affiliated to University of Mysore), Siddhartha Nagara, Mysore, Karnataka 570011, India

5 Department of Biochemistry and Molecular Biology, School of Life Sciences, Central South University, Changsha 410013, China

6 Department of Thoracic Surgery, The Third Affiliated Hospital of Southern Medical University, Guangzhou, China 
lungs, kidney and intestines. It is located on Xp22.2 and encoded an 805-amino-acid-long protein and belongs to the family of angiotensin-converting enzyme of dipeptidyl carboxydipeptidases $[1,2]$. ACE2 contains a 17-amino-acid $\mathrm{N}$-terminal signal peptide and a 22 -amino acid $\mathrm{C}$-terminal membrane anchor. It also has a conserved motif at the zinc metalloprotease consensus sequence (HEXXH) and a conserved glutamine residue at Glu-402 that served as a third zinc ligand [1]. ACE2 is also has homologous to the human ACE, which plays a role in cleaving angiotensin I into angiotensin 1-9, and vasoconstrictor angiotensin II peptide into the vasodilator angiotensin 1-7 [3, 4]. ACE2 correspondingly acts as the entry point into cells for several coronaviruses. By cleaving angiotensin II and increasing vasodilator angiotensin 1-7, it may act as an important regulator of heart function $[1,5]$ and plays a protective role in acute lung injury. The expression of ACE2 in organ- and cell-specific implies its regulatory role of cardiovasculature, kidneys and fertility. Potential anti-tumor effects of ACE2 and future therapeutic perspectives for cancers in ACE2 have been reported $[6,7]$. Unfortunately, ACE2 has a high affinity to severe acute respiratory syndrome coronavirus 2 (SARS-CoV-2). Importantly, ACE2 is a key host cell receptor for the spike (peplomer) glycoprotein of the coronavirus HCoV-NL63, also known as severe acute respiratory syndrome coronavirus (SARS-CoV) [8], and the very recently reported coronavirus disease 2019 (COVID-19) in Wuhan called severe acute respiratory syndrome coronavirus 2 (SARS-CoV-2) [9-11]. Diseases associated with the ACE2 gene include SARS, COVID-19, Middle East respiratory syndrome (MERS) and Hartnup disorder [10, 12, 13]. This might provide a clue that suppressing the expression levels of ACE2 in cells may help fight the viral infection.

Since its first report in December 2019 [14], the COVID19 is rapidly spreading worldwide and the number of cases is rising with increasing pace across all countries $[15,16]$. Due to its invasive spread, the World Health Organization (WHO) declared COVID-19 as a global pandemic on Mar. 11, 2020 (https://www.who.int/emergencies/diseases/novel -coronavirus-2019/events-as-they-happen) [17]. The onset of COVID-19 causes progressive respiratory failure and even death due to alveolar damage in lungs, because the virus enters host cells through the enzyme ACE2, which is expressed extremely highly in the type II alveolar cells of the lungs. As of the 20th of March 2020, the rate of deaths per number of identified cases is about $4.1 \%$ overall, ranging from 0.2 to $15 \%$ depending on age and other preliminary health issues. The binding affinity between ACE2 and SARS-CoV-2 is nearly 10 - to 20 -fold higher than that between ACE2 and SARS-CoV [18, 19]. Hence, in ACE2expressing cells and tissues, $A C E 2$ gene is the functional receptor of SARS-CoV-2 and plays a critical role in the viral entry into the cells during infection, and it also has potential anti-tumor roles in cancer. Therefore, in this study, we analyzed the expression profiles of the ACE2 gene for COVID-19 in different normal tissues and cancer tissues as a prognostic marker in renal and liver cancer.

\section{Materials and methods}

\section{Materials and machines}

Materials used in this study were RNAsimple Total RNA Kit (TIANGEN, Beijing, China), ReverTra Ace qPCR RT Master Mix (Cat No. FSQ-201, TOYOBO, China) [20]. Antibody for ACE2 was come from Sigma-Aldrich (HPA000288) or R\&D Systems (CAB026174) with dilution of 1:500. And the following machines used: Microscissor, tweezers, Benchtop centrifuge (Thermo Scientific), UV spectrophotometer, Veriti 96 well thermal cycler PCR (Applied Biosystems, USA).

\section{RNA-sequencing profiles}

In order to determine tissue-specificity, the ACE2 gene expression profiles were analyzed from RNA-sequencing (RNA-seq) data, performed on tissue samples from 95 human individuals representing 27 different tissues, using the NCBI program (https://www.ncbi.nlm.nih.gov/ gene/59272) [21]. These data were collected from RNAseq normal tissues (BioProject: PRJEB4337; Publication: PMID 24309898).

\section{Homology analysis}

Homologs of the ACE2 gene were performed by the NCBI program (https://www.ncbi.nlm.nih.gov/homologene?LinkN ame $=$ gene_homologene $\&$ from_uid=59272) $[20,22,23]$. Multiple sequence alignment was performed by Clustal Omega program.

\section{Total RNA extraction}

RNA extraction from mice tissues was executed according to our previously reported standard protocols [22, 24-26]. Tissues from 2 month old adult mice included retina, lung, uterus, ovary, breast, testis, spleen, kidney, liver, brain, small intestine, skeletal muscle, and whole blood. After extraction, $1 \mu \mathrm{L}$ of RNA sample was used to determine and record the concentration value using the UV spectrophotometer, before stored in a $-20{ }^{\circ} \mathrm{C}$ refrigerator [20]. 


\section{Reverse transcription (RT)}

Total RNA was transcribed into cDNA according to our previously described methods [24, 27]. The reverse transcription Polymerase Chain Reaction (RT-PCR) amplification reaction system was $20 \mu \mathrm{L}$, including $5 \times$ RT Master Mix $4 \mu \mathrm{L}$, RNA template $2 \mu \mathrm{g}$, and $\mathrm{ddH}_{2} \mathrm{O}$ was added to make the final volume $20 \mu \mathrm{L}$. The RT-PCR cycle was programmed as follows: $37^{\circ} \mathrm{C}$ for $15 \mathrm{~min}, 50{ }^{\circ} \mathrm{C}$ for $5 \mathrm{~min}$, then at $98^{\circ} \mathrm{C}$ for 5 min, and finally hold at $4{ }^{\circ} \mathrm{C}$. Then, the PCR product was then diluted six fold and stored at a $-20^{\circ} \mathrm{C}$ refrigerator, or used it directly in the next experiment.

\section{Semi-quantitative RT-polymerase chain reaction (Q-RT-PCR)}

Q-RT-PCR was conducted according to our previously reported protocols [22, 24]. Primers for the Ace2 gene (NM_001130513.1) in mouse were (RT-ACE2-mF: TCC ACGATCCCATGCCTATG; RT-ACE2-mR: TGCTGA AGACCCACTTTGCT). Mouse $\beta$-actin gene was used as an internal control [20,22]. Primers for RT-PCR spanned at least one intron to avoid amplification of genomic DNA. The reaction system used for PCR amplification was $10 \mu \mathrm{L}$, consisting of Ace 2 primers $1 \mu \mathrm{L}, 2 \times$ Taq PCR Master mix $5 \mu \mathrm{L}$, cDNA $1 \mu \mathrm{L}, \mathrm{ddH}_{2} \mathrm{O} 3 \mu \mathrm{L}$. PCR program was as follows: pre-denaturation at $95{ }^{\circ} \mathrm{C}$ for $90 \mathrm{~s}$, denaturation at $95{ }^{\circ} \mathrm{C}$ for $40 \mathrm{~s}$, annealing at $60^{\circ} \mathrm{C}$ for $30 \mathrm{~s}$, extension at $72{ }^{\circ} \mathrm{C}$ for $30 \mathrm{~s}$ for 31 cycles; final extension at $72{ }^{\circ} \mathrm{C}$ for $5 \mathrm{~min}$, and storage at $4{ }^{\circ} \mathrm{C}$. Each assay was performed as a triplicate. PCR products and $2 \mathrm{~kb}$ DNA molecular weight size ladder were loaded in a $1.8 \%$ agarose gel to be confirmed by electrophoresis [22].

\section{Immunohistochemistry expression profiles from normal and cancer tissues}

Immunohistochemistry (IHC) expression profiles of ACE2 for normal tissues of testis and lung were analyzed through the Human Protein Atlas (https://www.proteinatlas.org/ ENSG00000130234-ACE2/tissue). Pathological IHC, TCGA RNA samples expression profiles and survival analysis of ACE2 for the lung, renal and liver cancer tissues were analyzed in the Human Protein Atlas from renal cancer patient data (https://www.proteinatlas.org/ENSG0 0000130234-ACE2/pathology/renal+cancer) and liver
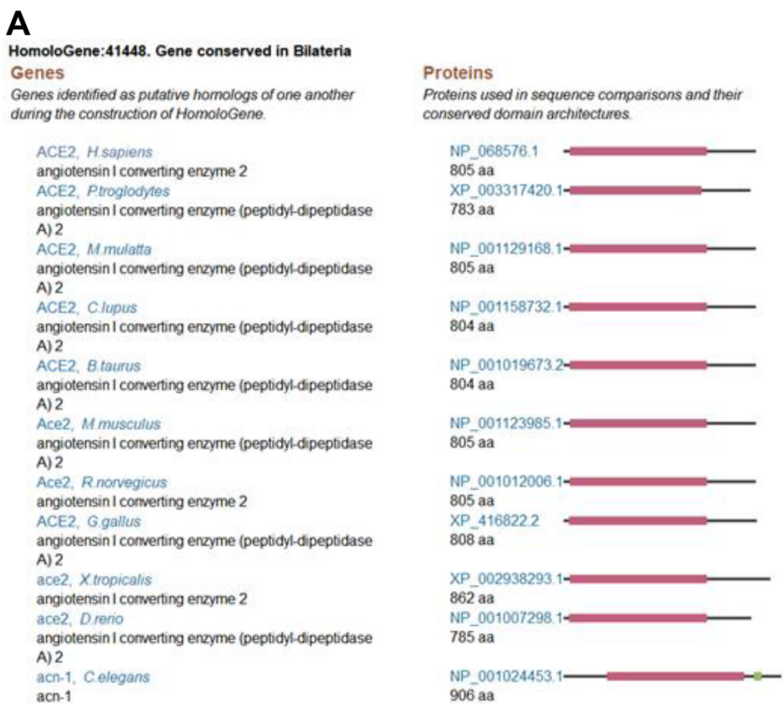

B

Conserved Domains

Conserved Domains from $C D D$ found in protein sequences

by rpsblast searching

EB (pfam01683)

프 EB module.

Gluzincin (cl14813)

= Peptidase Gluzincin family (thermolysin-like

proteinases, TLPs) includes peptidases $\mathrm{M} 1, \mathrm{M} 2, \mathrm{M} 3$

M4, M13, M32 and M36 (fungalysins)
C

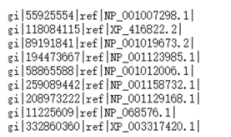

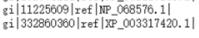

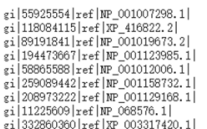

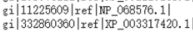

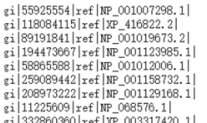

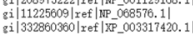

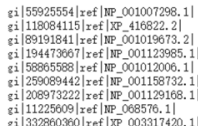

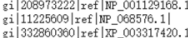

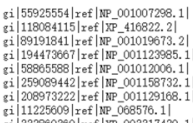

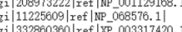

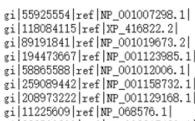

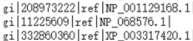

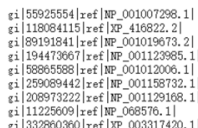

Fig. 1 Homologs of the ACE2 proteins. a Conservation for ACE2 in 11 different species. b The indicated two conserved domains. c Multiple sequence alignment results of the N-terminus of ACE2 (RBD located terminal) among indicated different species. "*” indicates the identity whereas "." and ":" show the similarity. Amino acids from 1 to 418 of human ACE2 are presented 
cancer patient data (https://www.proteinatlas.org/ENSG0 0000130234-ACE2/pathology/liver+cancer), respectively. The standard immunostaining protocol for IHC can be found at protocols.io (https://www.protocols.io/view/hpa-cell-atlas -standard-immunostaining-protocol-x2dfqa6). Normal tissues were represented by samples from three individuals each, using one core per individual; cancer tissues were sampled from two cores of each individual and protein expression was annotated in tumor cells. Specimens containing normal and cancer tissue were collected from anonymized paraffin-embedded material of surgical specimens. Note that samples used for IHC did not correspond to the samples in the TCGA dataset.

\section{Survival analysis in TCGA}

Patients were classified into two expression groups based on the value of FPKM (fragments per kilobase of exon model per million reads mapped) in each gene and the correlation between expression level and patient survival was examined. The Kaplan-Meier survival estimators were used to examine the prognosis of each group of patients, and the patient survival outcomes were compared by log-rank tests. The genes with log-rank $\mathrm{P}<0.001$ by Kaplan-Meier analysis were defined as a prognostic. If the group of patients with high expression of the prognostic gene had a higher observed event than expected, it was an unfavorable prognostic gene; otherwise, the results showed a favorable prognosis. Genes with a median expression less than FPKM value of 1 were minimally expressed and classified as unprognostic, even if they showed significant prognosis by survival analysis.

\section{Results}

\section{Determination of ACE2 conservation}

Homologs of the ACE2 protein demonstrated that it is highly conserved in different species, such as, chimpanzee, Rhesus monkey, cow, dog, mouse, rat, chicken, zebrafish, C. elegans, and frog (Fig. 1a, b). This suggested that ACE2 from these animals of different species could potentially bind the receptor binding domain (RBD) of the spike glycoprotein $[11,28]$, making them probable natural hosts for SARSCoV-2. Multiple sequence alignment of the N-terminus of ACE2 (RBD located terminal) among different species showed very high levels of identity and similarity in the amino acids (Fig. 1c).

\section{The mRNA expression profiles for ACE2 in human and Ace 2 in mouse}

The mRNA expression profiles for the ACE2 gene in humans were analyzed from RNA-sequencing data in the indicated
Fig. 2 The mRNA expression profiles in human and mouse. a The $A C E 2$ expression profiles in the indicated 27 different human tissues. RPKM stands for number reads per kilobase $(\mathrm{kB})$ of transcript per million mapped reads. In RNA-Sequencing, the relative expression of a transcript was proportional to the number of cDNA fragments that originate from it. $\mathbf{b}$ The Ace 2 gene mRNA expression profile analysis in the different tissues of organisms in the mouse. $\mathrm{NC}$ negative control, muscle skeletal muscle

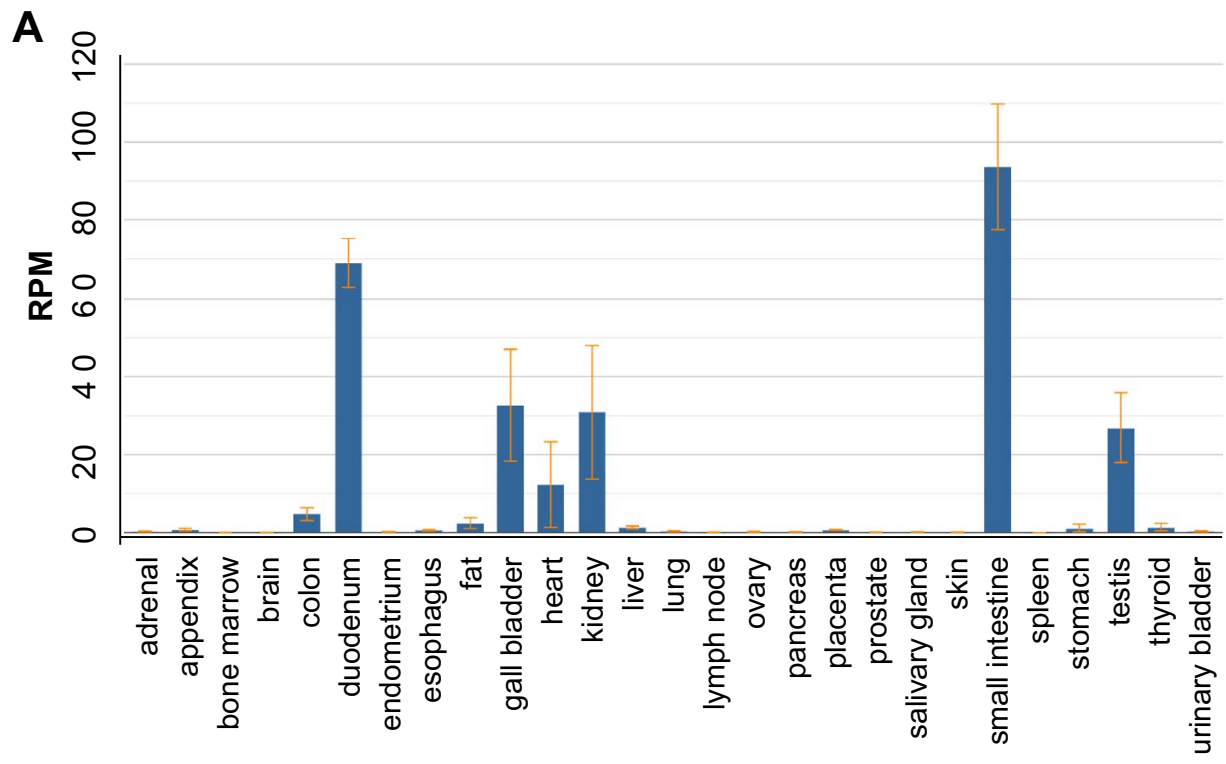

B

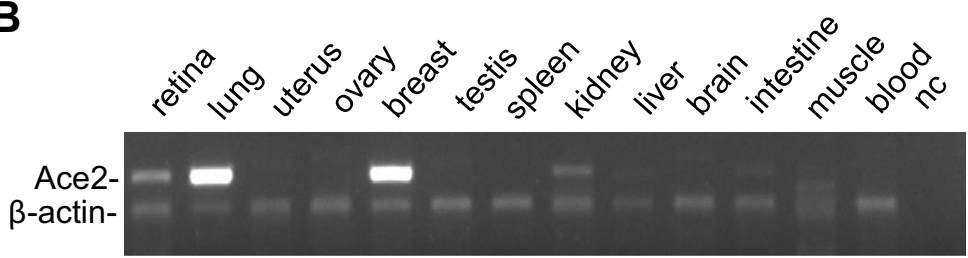


27 different human tissues. The RPKM (Reads Per Kilobase of transcript per Million mapped reads) values of ACE2 expression in the small intestine was found to be highest, with approximately 93.7, followed by the duodenum (69), gall bladder (32.6), kidney (30.8), testis (26.89), and heart (12.3), but it was found to be lowest in the spleen with approximately 0.007 (Fig. 2a, Table 1). Thus, these results showed the biased expression profile of ACE2 mRNA in the small intestine, duodenum and 5 other tissues.

Analysis of the expression profile of Ace 2 mRNA in the 13 indicated different tissues from mice was conducted by Q-RT-PCR (Fig. 2b). Surprisingly, the results showed that Ace 2 transcripts are highly expressed in lung and breast, lower in expression in the retina, kidney and small intestine, but very weakly expressed in the testis; where were no detectable expressions in other tissues we tested (Fig. 2b). Comprehensively, ACE2 expression distribution is organspecific, and it is mainly localized in the kidney, the cardiovascular and gastrointestinal systems, male testis and female breasts. The expression of ACE2 in different tissues suggests multiple routes of SARS-COV2 transmission.

\section{IHC analysis results in testis and lung tissues}

IHC analysis using the ACE2 antibody found selective membranous expression in renal tubules, intestinal tract, gallbladder and testis (Fig. 3, data not shown). In normal testis tissues, ACE2 presented high staining in cells of seminiferous ducts and leydig, located in both cytoplasmic and membranous region of the cells (Fig. 3a, b). In other male tissues, including ductus deferens, epididymis, seminal vesicle, and prostate, it was not detected or its expression is very low (data not are shown).

IHC analysis of normal lung tissue indicated that ACE2 expression in alveolar macrophages is moderate and is mainly located in both the cytoplasm and membrane (Fig. 3c, blue arrows). A few positive staining of ACE2 in type I alveolar epithelial cells (Fig. 3c, black arrow) and type II alveolar epithelial cells (Fig. 3c, red arrows) was also revealed; but most of type II alveolar epithelial cells were ACE2 negative stained (Fig. 3c, dashed red arrows).

\section{Expression analysis results in cancer tissues}

IHC analysis using the ACE2 antibody (CAB026174) indicated that renal cancers and a few cases of colorectal and liver cancers exhibited moderate to strong immunoreactivity in the membrane (Fig. 4a). The remaining cancer tissues generally did not exhibit expression (Fig. 4a).
Table 1 Expression of ACE2 mRNA in human different tissues

\begin{tabular}{|c|c|c|c|}
\hline Sample & Numbers & RPKM values & Counts \\
\hline Adrenal & 3 & $0.287 \pm 0.08$ & 15,093 \\
\hline Appendix & 3 & $0.751 \pm 0.291$ & 37,467 \\
\hline Bone marrow & 4 & $0.049 \pm 0.029$ & 6316 \\
\hline Brain & 3 & $0.045 \pm 0.023$ & 2583 \\
\hline Colon & 5 & $4.695 \pm 1.662$ & 583,961 \\
\hline Duodenum & 2 & $69.049 \pm 6.292$ & $2,262,846$ \\
\hline Endometrium & 3 & $0.2 \pm 0.055$ & 11,145 \\
\hline Esophagus & 3 & $0.589 \pm 0.17$ & 51,888 \\
\hline Fat & 3 & $2.389 \pm 1.378$ & 130,013 \\
\hline Gall bladder & 3 & $32.606 \pm 14.37$ & $2,936,794$ \\
\hline heart & 4 & $12.309 \pm 10.958$ & $1,505,105$ \\
\hline Kidney & 4 & $30.81 \pm 17.148$ & $2,168,804$ \\
\hline Liver & 3 & $1.294 \pm 0.368$ & 87,261 \\
\hline Lung & 5 & $0.345 \pm 0.102$ & 31,059 \\
\hline Lymph node & 5 & $0.063 \pm 0.071$ & 7576 \\
\hline Ovary & 2 & $0.194 \pm 0.106$ & 13,770 \\
\hline Pancreas & 2 & $0.199 \pm 0.029$ & 13,240 \\
\hline Placenta & 4 & $0.675 \pm 0.102$ & 83,518 \\
\hline Prostate & 4 & $0.099 \pm 0.037$ & 7124 \\
\hline salivary gland & 3 & $0.147 \pm 0.009$ & 16,539 \\
\hline Skin & 3 & $0.089 \pm 0.053$ & 8127 \\
\hline Small intestine & 4 & $93.724 \pm 16.1$ & $7,099,334$ \\
\hline Spleen & 4 & $0.007 \pm 0.003$ & 702 \\
\hline Stomach & 3 & $1.177 \pm 0.937$ & 96,543 \\
\hline Testis & 7 & $26.895 \pm 8.944$ & $6,060,081$ \\
\hline Thyroid & 4 & $1.392 \pm 0.928$ & 187,069 \\
\hline Urinary bladder & 2 & $0.33 \pm 0.107$ & 19,218 \\
\hline
\end{tabular}

TCGA dataset of the RNA-sequencing data from RNA cancer patients indicated that mRNA levels of ACE2 increased in renal cancer of 877 patients (Fig. 4b, average FPKM of 26.9 in renal cancer, and average FPKM of 2.3 in liver cancer).

\section{Survival analysis in cancer patients}

Given that our studies focused on ACE2 expression and showed that $A C E 2$ expressions are higher in both normal and cancer tissues of kidney and liver than that of lung tissues, further clinical relationships between the ACE2 expression and survival outcomes were also investigated. From the available data of renal and liver cancer patients, survival analysis was performed, and the renal and liver survival results are shown in Fig. 5. High expression of $A C E 2$ was correlated with long survival in renal cancer 

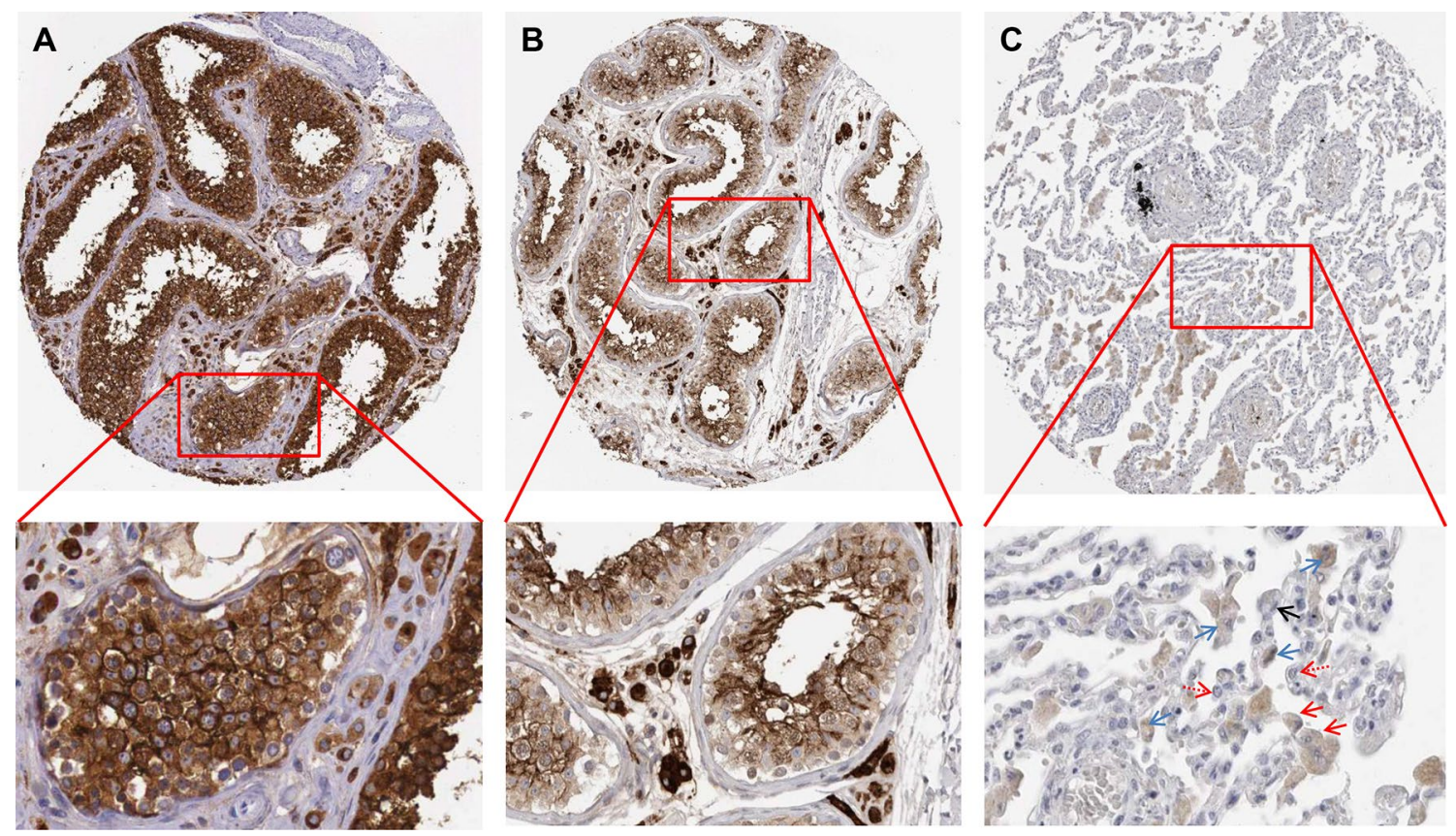

Fig. 3 Immunohistochemistry (IHC) images of normal tissues (testis and lung) for ACE2. a IHC image of normal testis tissue from a male of age 38 (Patient id: 305). b IHC image of normal testis tissue from a male of age 26 (Patient id: 2254). c IHC image of normal lung tissue from a female of age 49 (Patient id: 2268). Bottom panels, enlarged pictures from top panels. Blue arrows indicate the rep-

patients, with $75 \%$ high survival rate and $58 \%$ low survival rate respectively (Fig. 5a, P-value 0.000021); high expression of ACE2 was correlated with longer survival in 365 liver cancer patients, with $64 \%$ high survival rate and $37 \%$ low survival rate respectively (Fig. 5b, P-value 0.00042 ), indicating that $A C E 2$ is a prognostic marker in renal and liver cancers. Thus, high expression of $A C E 2$ is favorable for a higher 5-year survival rate of cancer patients in renal and liver cancer.

\section{Discussion}

In this study, we performed data analysis of RNA-sequencing from NCBI and quantitative RT-PCR from mouse, and found that $A C E 2$ expression distribution is organ-specific and mainly found in the kidney, male testis, female breast, and cardiovascular and gastrointestinal system. This finding is consistent to Harmer et al. reports, showing its highest expression is in the testis, renal tissues and cardiovascular tissues, and all portions of the gastrointestinal tract [29]. IHC analysis using ACE2 antibody in normal testis tissues showed that ACE2 is presented high staining in cells of seminiferous ducts and leydig. High level of ACE2 expression in the testis, cardiovascular and gastrointestinal resentative positive results in macrophages, black arrow indicates the representative positive results in type I alveolar epithelial cells, red arrows indicate the representative positive results in type II alveolar epithelial cells (c, red arrow), but dashed red arrows indicate the representative negative staining of type II alveolar epithelial cells. (Color figure online)

system indicated that SARS-CoV-2 might not only attack the lung, but also other organs namely the testis, and may affect sexual development for younger boys and cause infertility in adult males if they had been infected by the SARS-CoV-2. Surprisingly, the results showed high expression of Ace 2 transcript in the lungs of the mouse but low in humans. However, Itoyama et al. [2] identified an alternative 5-prime untranslated exon and found expression of the alternative 5-prime exon in various major organs including in the lungs, demonstrating that different Ace 2 transcripts existed. However, this does not discount the possibility that the ACE2 expression in the lungs of humans may truly be lower. With this regard, we looked at the expression levels by western blotting in human normal tissues, and we found that the ACE expression in the lungs is truly very low, comparing to the testis tissues (link here: https://www.abcam.com/ace2-antibody-ab153 48.html). Taken together, even though lung cells may be the main target cells of SARS-CoV-2, other organs may also be vulnerable [30], particularly in the testis which may lead to male infertility if he had COVID-19.

However, in the context of SARS-CoV-2 infection and cancer, is ACE2 friend or foe? Since ACE2 might play potential anti-tumor effects of and future therapy for cancer $[6,7]$, we analyzed $A C E 2$ expression and its clinical significance associated with survival rates of cancer patients. 


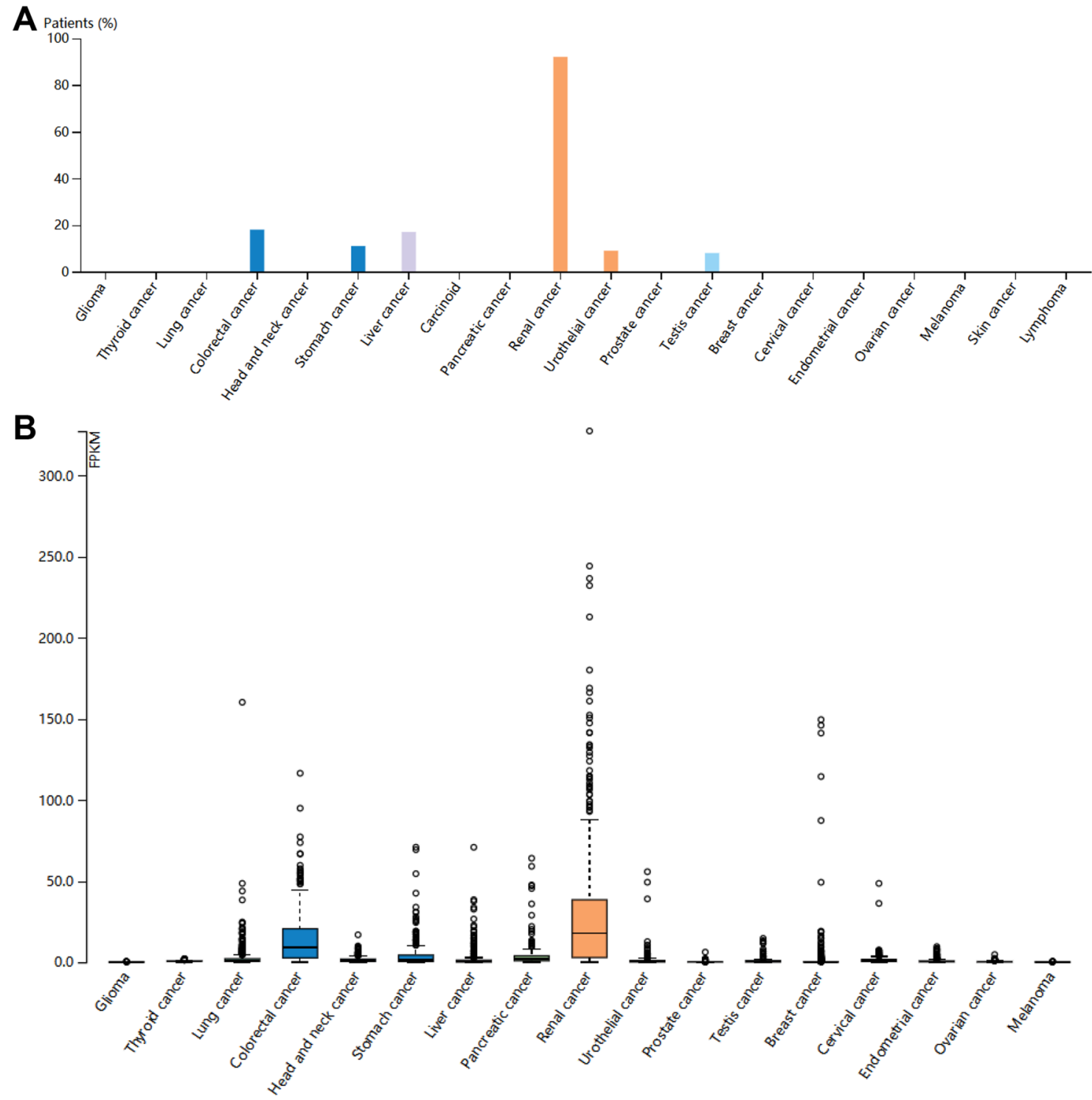

Fig. 4 The protein and mRNA expression overviews for ACE2 in humans. a The ACE2 protein expression overviews. Tissue microarrays are used to show antibody staining for IHC in 20 different types of cancer with total samples of 216 cancer patients. Color-coded bars indicate the percentage of patients with high and medium protein expression levels in each cancer. The types of cancer are color-coded according to which type of normal organ cancer originates from. ACE2 protein expression with low or un-detectable will results in a white bar. b The ACE2 mRNA expression overviews. RNA expres-

In cancer tissues, renal cancer and a few cases of colorectal and liver cancer exhibited moderate to strong membranous immunoreactivity to ACE2; the remaining cancer tissues were all generally negative. RNA-sequencing indicated that mRNA levels of $A C E 2$ were enhanced in renal and liver cancer. Further survival analyses showed that high expression of $A C E 2$ is correlated with longer survival in renal and sion overview shows RNA-seq data from The Cancer Genome Atlas (TCGA) from 17 cancer types representing 21 cancer subtypes with a corresponding major cancer type in the Human Pathology Atlas for comparisons. The FPKMs for each gene were subsequently used for quantification of expression with a detection threshold of the FPKM value of 1 . Genes were categorized using the same classification as described above. RPKM stands for number fragments per kilobase of exon per million reads

liver cancer, indicating that ACE2 is a prognostic marker in both renal cancer and liver cancer.

In conclusion, ACE2 expression distributions were organspecific, mainly in kidney, male testis, female breast, and the cardiovascular and gastrointestinal systems. Thus, SARSCoV-2 infection may most likely affect male infertility. On the other hand, high expression of ACE2 is correlated with 

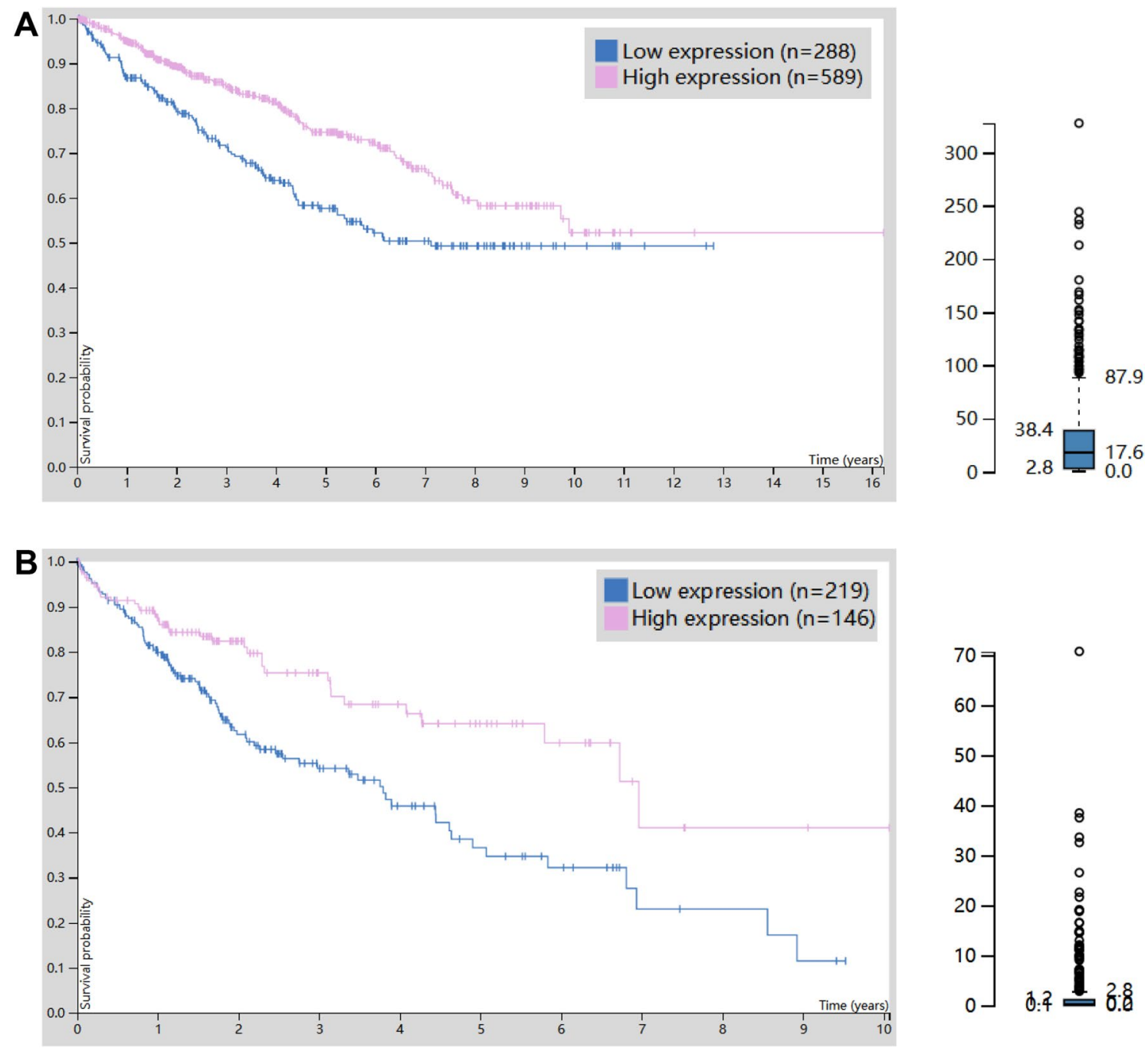

Fig. 5 Kaplan-Meier plots for all cancers where high expression of the ACE2 gene has a significant $(\mathrm{P}<0.001)$ association with patient survival are shown in this summary. Prognostic marker is indicated in renal cancer (favorable) and liver cancer (favorable). Whether the prognosis is favorable or unfavorable is indicated in brackets. a

long survival in renal and liver cancer, indicating that $A C E 2$ is a prognostic marker in both renal cancer and liver cancer. The binding affinity between ACE2 and SARS-CoV-2 is nearly 10- to 20-fold higher than that between ACE2 and SARS-CoV [18, 19]. Hence, ACE2-expressing cells and tissues can potentially act as targets of the novel coronavirus. With all this combined, ACE2 is a functional receptor for SARS-CoV-2 and has potential anti-tumor role in cancer and may pose conflicting medical benefits and concerns. Altogether, this study may not only provide potential clues for further clinical management of COVID-19 pathogenesis and possible male fertility, but it might also indicate the clinical significances of the ACE2 gene in mortality rates of certain cancers.
Kaplan-Meier plot includes ACE2 expression and survival data for patients with renal cancer. b Kaplan-Meier plot includes ACE2 expression and survival data for patients with liver cancer. Right panels indicate the TCGA RNA-seq dataset for renal and liver cancer respectively

Acknowledgements We appreciate people in our lab for technical assistance. We truly thank Shangyi (Shelly) Fu from University of Houston/Baylor College of Medicine for editing the manuscript.

Author contributions JF, XL conducted experiments. BZ, LZ, CW, $\mathrm{JC}, \mathrm{HC}, \mathrm{JF}$ collected and analyzed the data. JF and ZJ designed and supervised the project. JF wrote the manuscript. BK and JF edited the manuscript.

Funding This work was supported in part by the National Natural Science Foundation of China (81672887 and 30371493), and the Joint Research Foundation of Luzhou City and Southwest Medical University (2018LZXNYD-YL01). 


\section{Compliance with ethical standards}

Conflict of interest The authors declare that they have no conflict of interest.

Ethics approval Experiments involving mouse followed the international, national, and institutional guidelines for the care and use of laboratory animals. The study has the Ethical Committee approval granted by the Southwest Medical University. This article does not contain any studies with human participants performed by any of the authors.

\section{References}

1. Tipnis SR, Hooper NM, Hyde R, Karran E, Christie G, Turner AJ (2000) A human homolog of angiotensin-converting enzyme. Cloning and functional expression as a captopril-insensitive carboxypeptidase. J Biol Chem 275:33238-33243

2. Itoyama S, Keicho N, Hijikata M, Quy T, Phi NC, Long HT, Ha LD, Ban VV, Matsushita I, Yanai H, Kirikae F, Kirikae T, Kuratsuji T, Sasazuki T (2005) Identification of an alternative 5 '-untranslated exon and new polymorphisms of angiotensinconverting enzyme 2 gene: lack of association with SARS in the Vietnamese population. Am J Med Genet A 136:52-57

3. Boehm M, Nabel EG (2002) Angiotensin-converting enzyme 2: a new cardiac regulator. N Engl J Med 347:1795-1797

4. Crackower MA, Sarao R, Oudit GY, Yagil C, Kozieradzki I, Scanga SE, Oliveira-dos-Santos AJ, da Costa J, Zhang L, Pei Y, Scholey J, Ferrario CM, Manoukian AS, Chappell MC, Backx PH, Yagil Y, Penninger JM (2002) Angiotensin-converting enzyme 2 is an essential regulator of heart function. Nature 417:822-828

5. Donoghue M, Hsieh F, Baronas E, Godbout K, Gosselin M, Stagliano N, Donovan M, Woolf B, Robison K, Jeyaseelan R, Breitbart RE, Acton S (2000) A novel angiotensin-converting enzyme-related carboxypeptidase (ACE2) converts angiotensin I to angiotensin 1-9. Circ Res 87:E1-9

6. de Paula GA, Palmeira VA, Ribeiro TFS, Costa LB, de Sa Rodrigues KE, Simoes ESAC (2020) ACE2/angiotensin-(1-7)/mas receptor axis in human cancer: potential role for pediatric tumors. Curr Drug Targets. https://doi.org/10.2174/138945012166620 0210124217

7. Zhang Q, Lu S, Li T, Yu L, Zhang Y, Zeng H, Qian X, Bi J, Lin $Y$ (2019) ACE2 inhibits breast cancer angiogenesis via suppressing the VEGFa/VEGFR2/ERK pathway. J Exp Clin Cancer Res 38:173

8. Li W, Moore MJ, Vasilieva N, Sui J, Wong SK, Berne MA, Somasundaran M, Sullivan JL, Luzuriaga K, Greenough TC, Choe H, Farzan M (2003) Angiotensin-converting enzyme 2 is a functional receptor for the SARS coronavirus. Nature 426:450-454

9. Walls AC, Park YJ, Tortorici MA, Wall A, McGuire AT, Veesler D (2020) Structure, function, and antigenicity of the SARS-CoV-2 spike glycoprotein. Cell 181:281-292

10. Hoffmann M, Kleine-Weber H, Schroeder S, Kruger N, Herrler T, Erichsen S, Schiergens TS, Herrler G, Wu NH, Nitsche A, Muller MA, Drosten C, Pohlmann S (2020) SARS-CoV-2 cell entry depends on ACE2 and TMPRSS2 and is blocked by a clinically proven protease inhibitor. Cell 181:271-280
11. Yan R, Zhang Y, Li Y, Xia L, Guo Y, Zhou Q (2020) Structural basis for the recognition of SARS-CoV-2 by full-length human ACE2. Science 367:1444-1448

12. Guo YR, Cao QD, Hong ZS, Tan YY, Chen SD, Jin HJ, Tan KS, Wang DY, Yan Y (2020) The origin, transmission and clinical therapies on coronavirus disease 2019 (COVID-19) outbreak: an update on the status. Mil Med Res 7:11

13. Kowalczuk S, Broer A, Tietze N, Vanslambrouck JM, Rasko JE, Broer S (2008) A protein complex in the brush-border membrane explains a Hartnup disorder allele. FASEB J 22:2880-2887

14. Wang C, Horby PW, Hayden FG, Gao GF (2020) A novel coronavirus outbreak of global health concern. Lancet 395:470-473

15. Gentile I, Abenavoli L (2020) COVID-19: perspectives on the potential novel global threat. Rev Recent Clin Trials. https://doi. org/10.2174/1574887115999200228100745

16. Lai CC, Shih TP, Ko WC, Tang HJ, Hsueh PR (2020) Severe acute respiratory syndrome coronavirus 2 (SARS-CoV-2) and coronavirus disease-2019 (COVID-19): the epidemic and the challenges. Int J Antimicrob Agents 55:105924

17. Mahase E (2020) Covid-19: WHO declares pandemic because of "alarming levels" of spread, severity, and inaction. BMJ 368:m1036

18. Xia S, Liu M, Wang C, Xu W, Lan Q, Feng S, Qi F, Bao L, Du L, Liu S, Qin C, Sun F, Shi Z, Zhu Y, Jiang S, Lu L (2020) Inhibition of SARS-CoV-2 (previously 2019-nCoV) infection by a highly potent pan-coronavirus fusion inhibitor targeting its spike protein that harbors a high capacity to mediate membrane fusion. Cell Res 30:343-355

19. South AM, Tomlinson L, Edmonston D, Hiremath S, Sparks MA (2020) Controversies of renin-angiotensin system inhibition during the COVID-19 pandemic. Nat Rev Nephrol 1-3

20. Fu J, Cheng J, Zhou Q, Wei C, Chen H, Lv H, Fu J (2019) A novel missense variant c.G644A (p.G215E) of the RPGR gene in a Chinese family causes $\mathrm{X}$-linked retinitis pigmentosa. Biosci Rep 2:39

21. Zhou B, Wei C, Khan MA, Chen H, Fu J (2020) Correction to: Characterization and molecular cloning of novel isoforms of human spermatogenesis associated gene SPATA3. Mol Biol Rep 47:1551

22. Fu J, Cheng J, Liu X, Li J, Wei C, Zheng X, He T, Fu J (2018) Evaluation genotypes of cancer cell lines HCC1954 and $\mathrm{SiHa}$ by short tandem repeat (STR) analysis and DNA sequencing. Mol Biol Rep 45:2689-2695

23. Cheng J, Peng J, Fu J, Khan MA, Tan P, Wei C, Deng X, Chen H, Fu J (2020) Identification of a novel germline BRCA2 variant in a Chinese breast cancer family. J Cell Mol Med 24:1676-1683

24. Fu J, Zhang L, He T, Xiao X, Liu X, Wang L, Yang L, Yang M, Zhang T, Chen R, Xu J (2012) TWIST represses estrogen receptor-alpha expression by recruiting the NuRD protein complex in breast cancer cells. Int J Biol Sci 8:522-532

25. Cheng J, Fu J, Zhou Q, Xiang X, Wei C, Yang L, Fu S, Khan MA, Lv H, Fu J (2019) A novel splicing mutation in the PRPH2 gene causes autosomal dominant retinitis pigmentosa in a Chinese pedigree. J Cell Mol Med 23:3776-3780

26. Zhang L, Zhou Q, Cheng J, Khan MA, Fu J, Duan C, Sun S, Lv $\mathrm{H}, \mathrm{Fu} \mathbf{J}$ (2020) Targeted next-generation sequencing identified novel compound heterozygous variants in the $\mathrm{CDH} 23$ gene causing Usher syndrome type ID in a Chinese patient. Front Genet 9:128

27. Fu J, Qin L, He T, Qin J, Hong J, Wong J, Liao L, Xu J (2011) The TWIST/Mi2/NuRD protein complex and its essential role in cancer metastasis. Cell Res 21:275-289 
28. Chen Y, Guo Y, Pan Y, Zhao ZJ (2020) Structure analysis of the receptor binding of 2019-nCoV. Biochem Biophys Res Commun 525:135-140

29. Harmer D, Gilbert M, Borman R, Clark KL (2002) Quantitative mRNA expression profiling of ACE 2, a novel homologue of angiotensin converting enzyme. FEBS Lett 532:107-110

30. Zou X, Chen K, Zou J, Han P, Hao J, Han Z (2020) Singlecell RNA-seq data analysis on the receptor ACE2 expression reveals the potential risk of different human organs vulnerable to
2019-nCoV infection. Front Med. https://doi.org/10.1007/s1168 4-020-0754-0

Publisher's Note Springer Nature remains neutral with regard to jurisdictional claims in published maps and institutional affiliations. 